\section{E-Health Strategies}

Good information is the best medicine said dr.

Michael Debakey, but how to come to good information in healthcare? There is no lack of initiatives but they seem to be competitive in stead of collaborative. Communication and restoring confidence must prevent that the wheel is invented several times locally and good standards and standard systems have to be delivered centrally. There is no easy solution but it is possible to take small but firm steps in the good direction. There are five directions to go:

1. Create an e-health vision on (inter) national and regional level;

2. Share knowledge between healthcare organisations;

3. Improve co-operation by (tele)communication;

4. Agree on standards for defining and disseminating information;

5. Design new processes to recognise patient groups and optimise their care path.

There are some common threads running through these directions for management.

First there has to be a balance in national and regional development of ICT in healthcare. Too many initiatives strand because they do not move up or they cannot come down.

There is need for a strong commitment in a network of healthcare partners.

Secondly, the professional users have to be asked what relevance and which requirements

they really need. Too long IT suppliers have dropped their technology in healthcare

organisations without asking what was needed. The USIT model can help to give a prognosis of the success of an information system. Last but not least the walls between healthcare organisations have to be reshaped with the help of IT and the Internet in particular. A combination between the good old healthcare information systems, patient protocols and (bio)medical technology results will be the golden triangle in which the electronic patient record finally will succeed.

In this minitrack we see that culture in the management of e-health systems plays an important role if you want to change the strategy of the healthcare organization. The positive and negative attitude toward information systems will influence the use of the information systems. It is difficult to look into the future but still we take a look into the mobile and wireless solutions for e-health.

Benchmarking and learning from developments in other countries can speed up the progress.

The second part of this minitrack will deal with the discussion of evaluation of e-health which is not yet satisfactory in the healthcare organizations. The discussion will be about user satisfaction and acceptance but in connection with the workflow management minitrack also about relevance of information systems in healthcare.

\section{Ton AM Spil}

Faculty of Technology \& Management University of Twente

P.O. Box 217

7500 AE Enschede

The Netherlands

a.a.m.spil@sms.utwente.nl

Robert A. Stegwee

Faculty of Technology \& Management

University of Twente

P.O. Box 217

7500 AE Enschede

The Netherlands

r.a.stegwee@sms.utwente.nl 